\title{
A FUSION-BASED WORKFLOW FOR TURNING SLAM POINT CLOUDS AND FISHEYE DATA INTO TEXTURE-ENHANCED 3D MODELS
}

\author{
G. Sammartano ${ }^{1 *}$, A. Spanò ${ }^{1}$, L. Teppati Losè ${ }^{1}$ \\ ${ }^{1}$ LabG4CH, Department of Architecture and Design (DAD) - Politecnico di Torino Viale Mattioli 39, 10125 Torino (Italy) \\ (giulia.sammartano, antonia.spano, lorenzo.teppati)@polito.it
}

\section{Commission II}

KEY WORDS: mobile mapping, point clouds, SLAM, fisheye photogrammetry, image orientation, data fusion, low-cost sensor

\begin{abstract}
:
Mobile mapping systems are increasingly developing ad hoc solution and integrated approaches for rapid and accurate 3D digitization in different operating environments belonging to built heritage assets. The use of emerging compact, portable and low-cost solution for imaging and ranging well fits in the purposes of mapping complex indoor spaces especially for narrow and underground ones (tunnels, mines, caves and ancient spaces), that are very challenging contexts in which to experiment integrated technological solutions and tailored workflows. In these cases, the main key issues are generally the difficulty in the seamless positioning and the complete and successful metric-radiometric content association in metric surface, due to the reduced manoeuvring space and complex lighting conditions. The prevalent goals for which the 3D digitization could be conceived are, beyond the accurate metric documentation, the analysis of mutual relations of volumes in complex structures, the virtual reconstruction and navigation of spaces with reduced accessibility for dissemination aims. The new SLAM-based positioning solutions implemented in some recent portable systems for indoor/outdoor mapping are increasingly developing and favoured by geometric features extraction algorithms even in traveling through complex and irregular environments. In parallel, the possibility to exploit the advances in digital photogrammetry algorithms for image matching and dense reconstruction using action-cam, compact and fisheye cameras allows to deploy investigation solutions even in complex environments at first sight impossible to map by photogrammetric approach. Here within the F.I.N.E. benchmark in the site of the San Vigilio Castle (Bergamo) and the "nottole" tunnels, a fusion-based workflow is proposed. It is focused on the purposes of providing radiometrically enriched 3D data from the possibility to colourized ZEB point cloud and a textured mesh surfaces with an oriented image block, taking care of the time processing steps optimization.
\end{abstract}

\section{INTRODUCTION}

In the last years, the research connected with MMS (Mobile Mapping Systems) have faced several challenges and transformations, especially in the fields connected with the survey of built heritage assets. The operative limits of these systems were progressively pushed further and their use in complex and challenging environments was deepened in literature by several research groups both for image- and rangebased data (Mandelli, Fassi, Perfetti, \& Polari, 2017; Sun \& Zhang, 2019). With the complexification of scenarios types, many challenging variables can come out in methods applicability and reliability issues and a comparison test-case can be a useful to face all the topics related to a performance evaluation. In fact, many portable instruments are under development in current researches and are frequently experienced in 3D mapping applications for indoor spaces, especially for complex and narrow environments. Tunnels (Rodríguez-Gonzálvez, Nocerino, Menna, Minto, \& Remondino, 2015), mines, caves and ancient underground chambers (Dewez, Yart, Thuon, Pannet, \& Plat, 2017) are interesting opportunities to document unusual spaces and their spatial relationships with the built heritage thank to the portability of new imaging and ranging technologies, despite some crucial topics are still under investigation (di Filippo et al., 2018; Sammartano \& Spanò, 2018; Tucci, Visintini, Bonora, \& Parisi, 2018a). Moreover, the main key issues are generally the difficulty in the seamless positioning and the complete and successful metric-radiometric content association in metric surface, due to the reduced manoeuvring space and complex lighting conditions.

\subsection{Low-cost solution for rapid mapping approaches for the documentation of complex built heritage spaces}

Concerning the documentation of these types of assets several solutions were proposed, researched and tested: both related with image and range-based approaches. Nevertheless, the use of emerging low-cost solution well fit in these purposes, as compact and action-cam for the image-based approaches (Calantropio, Patrucco, Sammartano, \& Teppati Losè, 2018) and, parallelly, some commercial packages of handheld mobile scanners based on ranging sensors are today available on the market. These two different approaches have obviously different characteristics and present different issues from the operative point of view. In general terms, range-based mobile mapping systems still have weakness both in the economical sustainability and in the radiometric information capturing and association. On the other side, the photogrammetric image-based reconstruction, based on camera orientation and dense matching, are more competitive in a general cost-benefit analysis but nevertheless a lot of research topics are still open on low-cost camera performance and calibration, multi-camera synchronization, etc.

The computed point clouds and the 3D textured surfaces derived from the acquisitions performed with these sensors offer the chance to perform a multi-scale and multi-content modelling in which firstly analyse, with continuous and comprehensive survey, the geometric relations among contiguous volumes at different levels, in a more rapid and intuitive way.

In the sector of range-based sensors the most recent and performing technological commercial solutions are multi-sensor high-performance platforms fully equipped with IMU (Inertial

Corresponding author 
Measuring Unit), ranging sensor coupled with calibrated cameras, single or multiple rig or with $360^{\circ}$ view too (Leica Pegasus Backpack, Kaarta Stencil), at the expense of the sustained costs. In case of multi-sensors platform developed with single camera equipment (e.g. Zeb REVO by GeoSLAM) the process of radiometric data association is under development and the final product is still lacking a complete and high-definition RGB data. It would require a separated photogrammetric acquisition with high resolution sensor that led to a data integration or more, as in the proposed approach, to a fusionbased data processing experimental method.

Particularly, the presented research is focused on proposing a stand-alone operative workflow to compute a fusion-based 3D model of a portion from the San Vigilio Castle with annex tunnel spaces derived from the joint use of the MMS and the fisheye photogrammetric data. The proposed approach aims to balance strengths and weaknesses from both approaches and to gain the benefit from the sensor's main contents (geometric definition, from portable SLAM (Simultaneous Localization And Mapping)-based MMS and radiometric content, from photogrammetric dataset), taking advantages from the individual time-cost allocation.

\section{THE F.I.N.E. BENCHMARK EXPLORATION}

In order to test the performances and feasibility of different survey approaches for such complex indoor mapping and for delivering a complete and continuous texture-enhanced 3D model, the F.I.N.E. (Fisheye Indoor Narrow spaces Evaluation) Benchmark is faced up (http://www.3d-arch.org/FINEbenchmark.pdf) (Bakuła, Mills, \& Remondino, 2019). In fact, the proposed research uses part of the benchmark dataset and is based on the integration of digital photogrammetry approach and mobile laser scanning 3D data in the site of the San Vigilio Castle (Bergamo, IT). The Castle tower, focus of the benchmark, is developed along three levels of spaces above ground, almost $15 \mathrm{~m}$ height, and an underground tunnel almost $100 \mathrm{~m}$ length (Figure 1). The setting up of an adjusted topographic network made by $\mathrm{n}^{\circ} 9$ vertices distributed along the Castle indoor and outdoor surveyed areas ensured the metric control of the other acquisitions and results and was used for the ZEB reference points measurements (Figure 2).

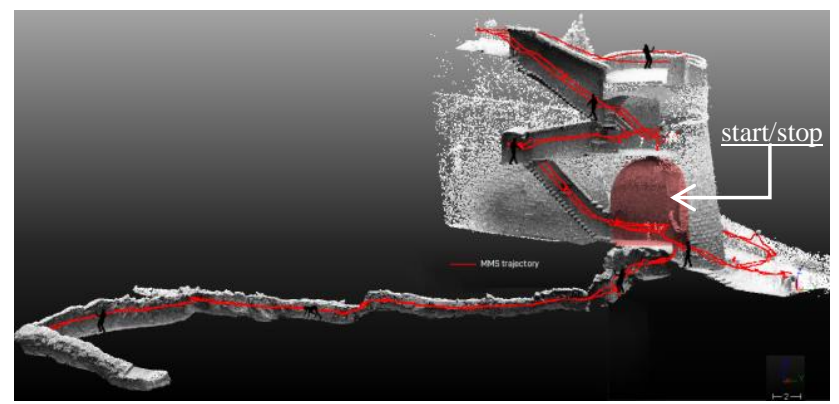

Figure 1. The three-levels tower and the underground "nottola" of the S. Vigilio Castel. The two main datasets and trajectories in red line and in red colour the entrance, as the area in common between the two datasets

However, the possibility to configure the network also inside along the underground "nottola" tunnel was very complex and it could not be accommodated. Thus, the reference ground-truth for the accuracy control of the proposed 3D reconstruction, in underground as well as in the tower floors, is a Leica RTC LiDAR (Light Detection And Ranging) points cloud. Furthermore, another range-based data was collected both in the tower and in the tunnel by means of a SLAM-based MMS, the
Zeb REVO-RT portable scanner. Two distinct photogrammetric datasets are proposed: the multi-camera rig and the fisheye single camera images. Here, the SLAM-based scans, with the help of the oriented fisheye block have been used in this experimental workflow. The accuracy control in the geometric reconstruction will be performed with the static LiDAR reference surface.

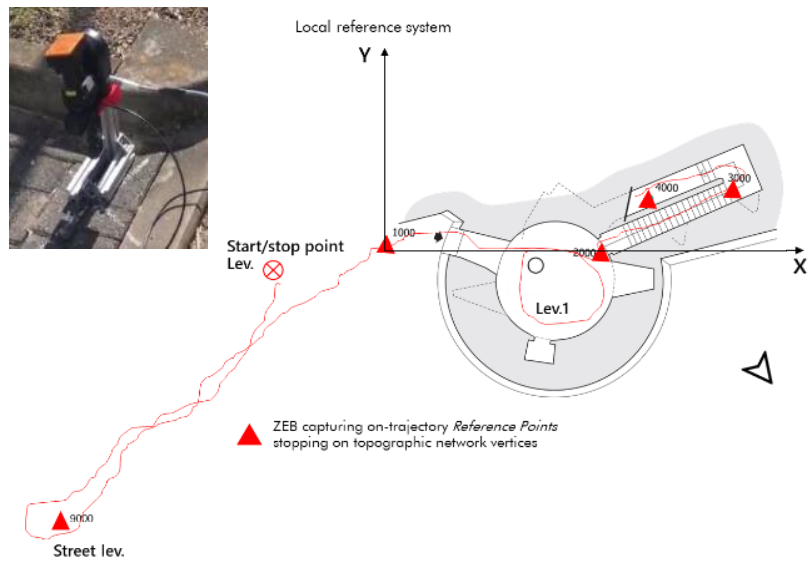

Figure 2. A sample of the topographic network vertices positions at ground and $1^{\text {st }}$ level, and the SLAM-based path mapping the reference points on the same vertices.

\subsection{Zeb REVO-RT system}

Nowadays, new SLAM-based positioning algorithms are implemented in portable systems for indoor/outdoor spaces mapping, equipping trolley, backpack or handheld devices, as the case of ZEB systems, from the Zebedee prototype up to the Zeb $R E V O-R T$ last release employed in this test (Bosse, Zlot, \& Flick, 2012; Cadge, 2016). The system, Figure 3 is now composed by a data logger with battery, linked to a rotating head carried by the operator hand, acquiring the LiDAR data with an Hokuyo ULM30LX laser, coupled with IMU sensor, a triaxial gyros, accelerometers and magnetometer. No intensity data is recorded.
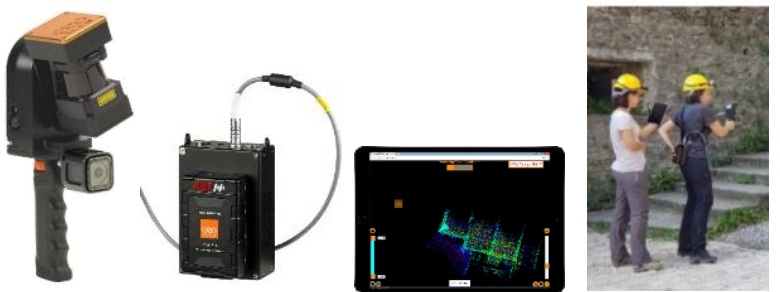

Figure 3. The Zeb REVO RT system and an image of the acquisition phase

\begin{tabular}{|ll|}
\hline \multicolumn{2}{|c|}{ Zeb REVO-RT } \\
\hline Wavelength & $905 \mathrm{~nm}$ \\
Eye-safe laser & Class 1 \\
Laser speed & $100 \mathrm{~Hz}$ \\
Laser lines & 100 lines $/ \mathrm{sec}$ \\
Scan speed & $x 2.5$ \\
Maximum range & $15-30 \mathrm{~m}$ \\
Points density & $\sim 43,200 \mathrm{pps}$ \\
3D Measurement declared accuracy & $+/-0.1 \%$ \\
FoV & $270^{\circ} \mathrm{H}_{\mathrm{FOV}} / 100^{\circ} \mathrm{V}_{\mathrm{FOV}}$ \\
Weight/portability (Head+data logger) & $\sim 2 \mathrm{~kg}$ \\
\hline
\end{tabular}

The solution of the positioning problem, without the use of GNSS, is based on trajectory estimation thanks to SLAM algorithms using both laser profiles data matching and 
positioning data as angular velocities combined with linear accelerations measurements (Nocerino, Menna, Remondino, Toschi, \& Rodríguez-Gonzálvez, 2017). The main specifications of the system are reported in the following Table 1.

The SLAM successful operation works with the consecutive alignment of the extracted profiles belonging to the environments and is favoured by geometric features extraction in traveling through consecutive environments gradually run across, better if complex or with articulated and irregular surfaces (Søren Riisgaard \& Morten Rufus Blas, 2005).

During the acquisition, the raw laser profiles continuously captured into time-windowed segments are rapidly and progressively re-projected in the real-time 3D reconstruction of spaces during the operator walking, according to the best correspondence to surface characterization, using an ICP-like approach for profile matching, with a global accuracy of the final processed point cloud identified with the relative accuracy declared by manufacturer of about 1-3 cm environment dependent. Many researches demonstrated increasing performances in indoor environments, between $3-5 \mathrm{~cm}$ errors deviation form a reference measure and $10-15 \mathrm{~cm}$ for respectively indoor and outdoor spaces. The main issues talked in the common approaches to the system assessment in the 3D mapping is the drift error depending from the trajectory length and progress, as well as the noise error function of the Lidar length distance, and the decreasing precision of laser profile increasing distance to the scanner (Eyre, Wetherelt, \& Coggan, 2016; Nocerino et al., 2017). Performance improvement occurs, as verified, with geometric features rich spaces, closed loops trajectories, constant and moderate walking speed $(<0.5 \mathrm{~m} / \mathrm{s})$ and still objects in the scene (Sammartano \& Spanò, 2018). In multi-level spaces, the importance of scans with overlapping spaces in common is crucial, namely vertical stairs blocks (Chiabrando, Sammartano, Spanò, \& Spreafico, 2019). An important evaluation should consider a local performance in the 3D surface digitization, with a over-all average value of $1000 \mathrm{pt} / \mathrm{mq}$ density and few centimetres precision in detail description (Tucci, Visintini, Bonora, \& Parisi, 2018b).

\subsubsection{SLAM-based point cloud acquisition with Zeb REVO-RT}

As introduced, a standard procedure of acquisition is proposed and followed. The environmental condition of the proposed case study well fit with the SLAM-based algorithm implemented in the sensor system: rich geometric constraints are distributed both in the tower floors and in the tunnel, whereas the outdoor space around the tower entrance and boundary walls as well as the top garden are large or regular spaces where no features enough occur to be detected. Different acquisition solutions have been tested, also due to failure problems sometimes occurring during the capturing phases related to the $\mathrm{Wi}-\mathrm{Fi}$ signal connection running between tablet and data logger. Nevertheless, the different configuration are referred to the two main parts of the study that have been mapped with roundtrip trajectories as in Figure 1: the tower and the tunnel.

According to setting condition, the path length and trend and the speed, the SLAM working was not well ensured and closed in all the six acquisition carried out. It is important to underline the challenging setting condition of scanning for ZEB Revo in this benchmark, above all for the tunnel entrance through a narrow manhole, and therefore in the state of a sudden geometry change. Finally, three main Zeb dataset were correctly acquired and are synthetized in (Table 2): aside from the two main parts of the building, the third scan was performed in order to finalise the test on the georeferencing procedure (next paragraphs).
The system implementation allows to rototranslate the Zeb point cloud using known coordinates points in order to (geo)reference the data in an established reference system. The solution is based on topographic vertices measurement as a set of reference points in order to check planimetric and vertical drift of the SLAM point cloud.

Some specificity of the Zeb point clouds are the added data that could enrich the point cloud geometry as: the SLAM-condition information (i.e. if SLAM profiles positioning works well according to the space conditions), the time attribute (i.e. the time progression of the measured profiles related to the trajectory), the geometry curvature value (i.e. the local orientation of the normal vectors of the point cloud).

\begin{tabular}{|l|c|cc|c|}
\hline \multicolumn{1}{|c|}{ TEST } & $\mathbf{N}^{\circ}$ points & Time & $\begin{array}{c}\text { Trajectory } \\
\text { length }\end{array}$ & $\begin{array}{c}\text { Average } \\
\text { speed }\end{array}$ \\
\hline 1 Tower & 27.289 .475 & $\begin{array}{l}14.9 \\
\mathrm{~min}\end{array}$ & $450 \mathrm{~m}$ & $\begin{array}{c}0.50 \\
\mathrm{~m} / \mathrm{sec}\end{array}$ \\
2 Tunnel & 25.638 .294 & $\begin{array}{l}14.6 \\
\mathrm{~min}\end{array}$ & $340 \mathrm{~m}$ & $\begin{array}{c}0.39 \\
\mathrm{~m} / \mathrm{sec} \\
0.38 \\
\mathrm{~m} / \mathrm{sec}\end{array}$ \\
$\begin{array}{c}\text { Reference } \\
\text { points }\end{array}$ & 15.280 .422 & $8.5 \mathrm{~min}$ & $196 \mathrm{~m}$ & $\mathrm{~m}$ \\
\hline
\end{tabular}

Table 2. San Vigilio SLAM-based datasets

A first evaluation on the acquisition results in this kind of complex environments comes out during the real-time visualization and after the scan closure operation just in the fieldwork, thanks to the navigation windows on the coupled mobile device. In fact, a preliminary and local rough profiles alignment is performed during the progression of acquisition, and a first processing of the SLAM computation is performed at the loop closure. It may happen indeed that the quality of closure is not correct and errors can occur in poorly featured and regular environments, in presence of moving objects such as people walking or closed/open doors, or in transitioning between environments, when the scene suddenly change, and the SLAM algorithm may have difficulty placing the new environment relative to the previous one. All these may cause bad positioning estimation for the trajectory points and a local or global SLAM error. An example of a trajectory alignment drift error than occurred in the tunnel scan is showed in Figure 4: starting and closing point do not correspond as well as the outward and return path along the tunnel that are duplicated geometries.

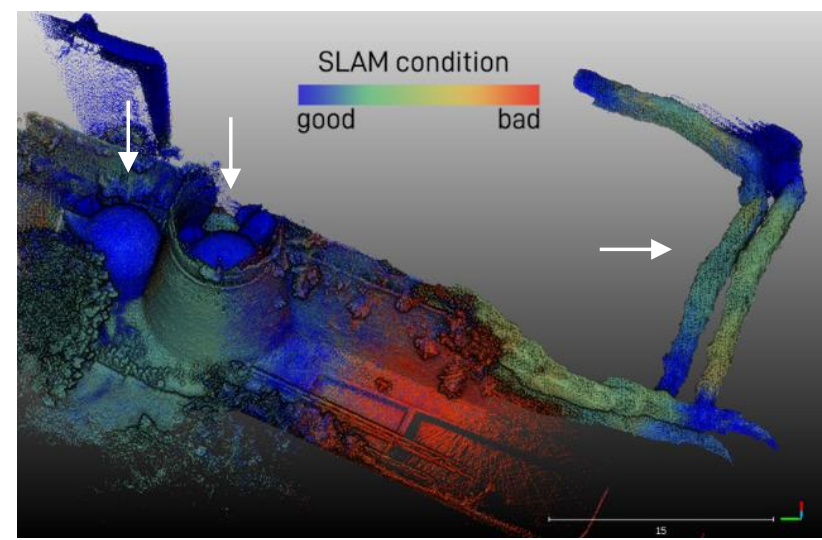

Figure 4. Example of a trajectory alignment drift error

\subsection{Fisheye-lens camera dataset}

One of the datasets that was distributed for the FINE benchmark consisted in a set of images acquired with a DSLR (Digital Single Lens Reflex) camera equipped with an $8 \mathrm{~mm}$ equisolid fisheye. 
The camera used was a Canon 5D MKIII equipped with a fisheye lens Sigma $8 \mathrm{~mm} \mathrm{f} / 3.5$ EX DG. A sample image of the dataset is reported in the following

Figure 5, while the main camera and lens specifications are reported in the following Table 3.

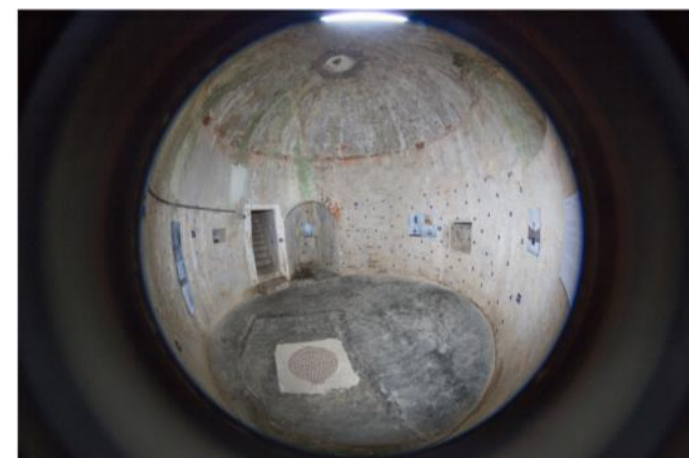

Figure 5. Sample image of the dataset acquired with the fisheyelens-equipped DSLR camera

\begin{tabular}{|cc|cc|}
\hline \multicolumn{2}{|c|}{ Camera } & \multicolumn{2}{c|}{ Lens } \\
\hline Sensor & Image Size & Lens focal & FoV \\
CMOS 22.3 MP & $5760 \times 3840$ & $8 \mathrm{~mm}$ & $180^{\circ}$ \\
\hline
\end{tabular}

Table 3. Essential camera and lens specifications of the fisheye dataset

Three datasets were acquired with this camera-lens set up and were then distributed in the framework of the FINE benchmark experience. The first dataset was dedicated on the underground tunnel, the second one to the interior of the tower and finally the third one for camera calibration. For the test presented in this paper, only the tower dataset was considered.

\subsubsection{Photogrammetric block first processing}

In general terms, the photogrammetric processing of these kind of images presents several issues, especially if the aim is to achieve an accurate metrical reconstruction of the surveyed object (Barazzetti, Previtali, \& Roncoroni, 2017; Perfetti, Polari, \& Fassi, 2018). Several problems must be considered, and different topic must be deepened. However, the focus of this research was slightly different: the main idea was to achieve a rapid, and possibly accurate, estimation of the camera positions in order to use this information to transfer the RGB information to the 3D model acquired with the SLAM approach. It was thus decided not to deepen other issues connected with the use of these data, but to focus more on the possibility of creating a pipeline to perform the fusion between the two approaches. For these reasons, the estimation of the I.O. parameters of this camera was solved adopting a self-calibration approach and using a set of control points. As will be further reported, two different set of control points were used to complete the estimation of the camera position and orientation: a set of topographic measured points (provided within the benchmark data) and a set of point which coordinates were extracted from the processed SLAM point cloud. These two different approaches were thus used to solve the orientation phase of the photogrammetric processing and the results of these two approaches are reported briefly in the next sections.

\section{THE SLAM-BASED POINT CLOUD WORKFLOW}

A complete workflow is proposed here in (Figure 6) and defined in order to finalize an optimization of the Zeb REVO-RT SLAMbased clouds. It is possible to delineate it with standard steps due to multiple research experiences and tests carried out by the authors research group in last few years and partially presented in Sammartano \& Spanò, 2018; Chiabrando et al., 2019; Chiabrando, Sammartano, \& Spanò, 2017. After the acquisition phase, even if collected with the best practice procedure as presented in the previous paragraph, it may happen that the raw point clouds need to be improved; to be corrected if eventually closed loops paths suffered from misalignments and drift errors occurred; to be gathered all together if a large area has been covered by multiple scans. These operations should be carried out in order to finalize an optimization of the most accurate Zeb SLAM-based point clouds.

The pipeline can be divided in: processing, optimisation of the first point cloud; metric evaluation of the results, re-processing if necessary; merging with non-rigid transformation; positioning with rigid roto-traslation for a relative or absolute positioning.

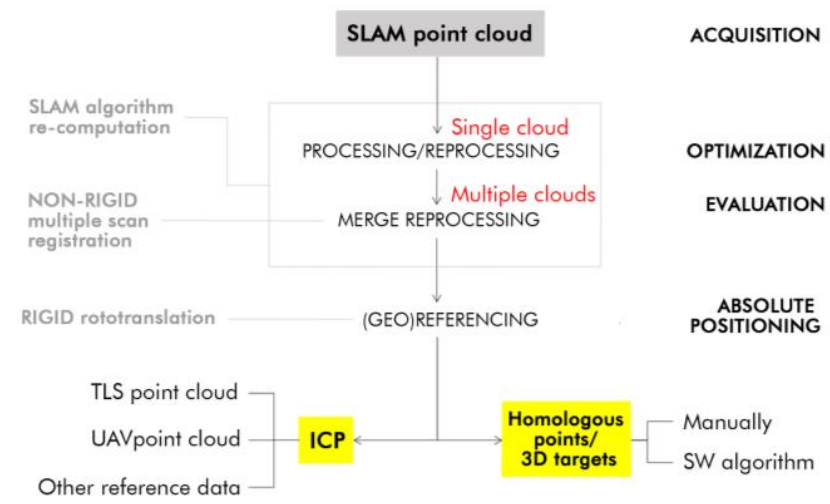

Figure 6. The workflow for ZEB data processing, evaluation, optimization and positioning

In case of re-processing, the algorithms work by means of the GeoSLAM Hub interface, and can be based on IMU and SLAM control and mutual balancing, together with other specific parameters dedicated to the improving of geometric shape recognition and noise reduction. A key role is assumed by the merge phase applied to multiple point clouds. In fact, multiple scans can be combined and spatially related with a merging process that can act not only as an alignment operation, but is a re-computation of the SLAM algorithm on each scans with nonrigid transformation. It allows improving of the scans using the reference shape of another contiguous cloud: this is the reason why is always necessary and warmly recommended too to have overlapping areas between contiguous scans.

\begin{tabular}{|l|cc|}
\hline \multirow{2}{*}{ Compared datasets } & \multicolumn{2}{|c|}{ Deviation analysis } \\
\cline { 2 - 3 } & Mean error (m) & St.dev. $(\mathrm{m})$ \\
\hline tower merged - non merged & 0,057 & 0,101 \\
tunnel merged - not merged & 0,037 & 0,075 \\
tunnel+tower merged - non & 0,157 & 0,218 \\
\hline
\end{tabular}

Table 4. Deviation analysis with merge processing

The final point cloud result benefits from the merge operation applied to these two scans, that shared the entrance area (Figure 1), allowing the merging procedure itself. The singular comparison between the two scans declare a significant reduction of the mean error vector calculation (Table 4) in the deviation analysis before and after the merging: a difference of about 5,7 $\mathrm{cm}$ in the tower dataset, and about $3,7 \mathrm{~cm}$ in the tunnel dataset. Considering the whole dataset, the maximum deviation is $15 \mathrm{~cm}$. This first analysis is only related to the effects of the merge function on the point cloud itself, without considering the ground-truth surface yet. 


\subsection{Geometry data analysis and evaluation}

In order to perform now a deeper evaluation of ZEB scans accuracy, a global evaluation of the point cloud acquired should be firstly conducted with a ground-truth surface (3.1.1), also due to the usual risk of being subject to drift error along significant trajectories distances. Here the LiDAR point cloud derived from the above mentioned RTC Leica scanner is considered for the benchmark as a reference surface despite the topographic control didn't reach the internal spaces of the underground tunnel. The LiDAR point cloud acquired and processed by FINE benchmark organization group, is composed by a total amount of 116 scans collected at almost $1 \mathrm{~m}$ mutual distance. The registration was based on $n^{\circ} 303 \mathrm{D}$ spherical targets and the global accuracy control delivered a final error on markers of about $4 \mathrm{~mm}$.

Then, due to the nature of the SLAM point clouds and their different behaviour in a local or global perspective, both the validations are considered, and a local evaluation of geometric reconstruction in the level of detail should be conducted too (3.1.2). It is quite well-known in researches that better performances of the Zeb REVO system evidently are retrieved if the validation is focused locally more than in the global 3D reconstruction.

3.1.1 Global point cloud assessment. In order to perform the first-step global evaluation of the 3D point cloud, both the separate (tower, tunnel) and joined parts (complete point cloud) are considered, and 3-steps of alignment solutions are proposed. The reason is related to the importance to take into account the partial and global verification of quality for the single scan by reprocessing the SLAM-algorithm and then with the whole multiple clouds registration.

\begin{tabular}{clccc}
\hline & \multicolumn{3}{c}{ ZEB clouds validated with RTC } \\
\cline { 2 - 4 } Test & \multicolumn{3}{c}{ Tunnel } & \multicolumn{3}{c}{ Tower } \\
$\mathbf{1}$ & Mean & 0,093 & Mean & 0,082 \\
& Dev.st. & 0,131 & Dev.st. & 0,121 \\
\hline \multirow{3}{*}{ Test } & Merged ZEB clouds validated with RTC \\
\cline { 2 - 4 } $\mathbf{2}$ & Tunnel & \multicolumn{3}{c}{ Tower } \\
& Mean & 0,090 & Mean & 0,081 \\
& Dev.st. & 0,126 & Dev.st. & 0,119 \\
\hline \multirow{3}{*}{ Test } & \multicolumn{4}{c}{ Validation alignment fixed in start point $\left(\mathrm{n}^{\circ} 10 \mathrm{CP}\right)$} \\
\cline { 2 - 4 } $\mathbf{3}$ & \multicolumn{3}{c}{ Complete cloud } \\
\multicolumn{4}{c}{ Mean } & 0,040 \\
\hline
\end{tabular}

Table 5. 3-steps global evaluation

1. ZEB clouds alignment and control with RTC. The first validation procedure takes into account the two separated raw point clouds compared to the reference surface. A deviation analysis is performed, delivering error results approximatively analogous, due to the high noise and variability of geometric objects involved in the tower indoor spaces and in the tunnel space: $M_{\text {error }}=8-9 \mathrm{~cm}$ with variability of st.dev $=12-13 \mathrm{~cm}$

2. Merged ZEB clouds alignment and control with RTC. After the merging step, that is the non-rigid alignment of the two scans, it can be useful to check if significant changes to the geometry happens in order to improve the shape due to possible drift error. Since this control is made by deviation analysis performed to the whole shape, it is well-known that the risk of distribution/reduction of error exists with the research of the best fitting adaptation between the two 3D surfaces, and no noteworthy decreasing of the error values from test 1 with this second solution occurs.
3. Alignment performed using $n^{\circ} 10$ Control Points fixing the starting area (Tower) and control with RTC. For this reason, a more rigid solution is applied to verify the actual error from the processed point clouds to the reference surface in order to asses any drift error. Thus, a set of $n^{\circ} 10$ points are used in the staring area to fix the point clouds and the deviation error is tested at the opposite side, at the end of the "nottola". In this case a better value of $4 \mathrm{~cm}$ of mean deviation error is extracted from the analysis, with a $S t \cdot d e v=2 \mathrm{~cm}$.

3.1.2 Local geometry evaluation. As expected the problem of trajectory drift error affected the point cloud in the global performance evaluation, but if a single object, i.e. the entrance room, is considered, the results of a comparison between the RTC Leica and the Zeb REVO point cloud give much better feedback. In fact, a 3D surface deviation analysis with ground truth show a small mean error $=0.012 \mathrm{~m}$ with a St. dev.: $0.016 \mathrm{~m}$. Looking also to the profile comparison and analysis show in Figure 7 is clearly visible the very noticeable $3 \mathrm{D}$ descriptive capability of the SLAM-based point clouds: $87 \%$ of digitized profile line deviate from the reference measure within $1,5 \mathrm{~cm}$.
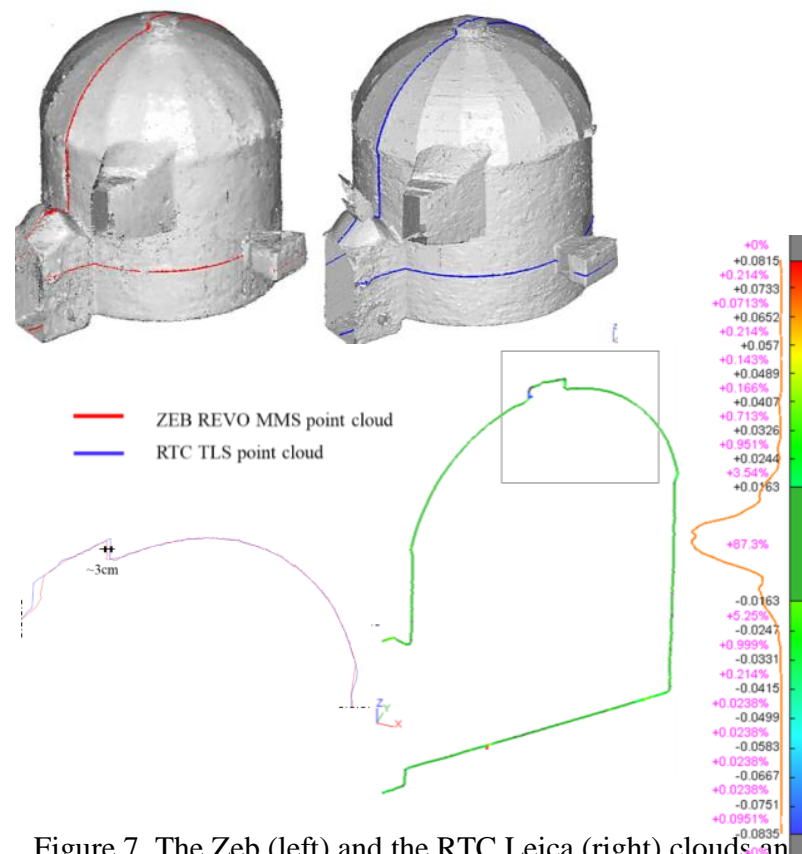

Figure 7. The Zeb (left) and the RTC Leica (right) cloudsans its features. Comparison of a sample profile section defining the accessible level of detail.

\subsection{The positioning problem}

During the acquisition it is possible to register with the Zeb REVO-RT trajectory a set of distributed points, i.e. topographic vertices, stopping with ZEB Revo RT instrument on the point for at least 10 seconds. This implementation has been tested and verifies in the benchmark, having at disposal the coordinates of the topographic network vertices.

The points coordinates, in the local reference system of ZEB Revo acquisition, are extracted during the processing and encrypted in the trajectory file. During the data reprocessing phase, the points list is generated in *.txt file containing "name, $X, Y, Z$, time, duration". $Z$ shift from the rotating head centre and the ground level point has to be considered.

A first step of accuracy control is made simply with the comparison of elevation values trend and discrepancies between the two measurements techniques (Table 6). The table reports the maximum error values in the central part of the tower elevation, along the stairs, where the V3000 and V400 were located. 


\begin{tabular}{|c|c|c|c|}
\hline & Topographic elev. & ZEB ref. elev. & $\Delta$ elev. \\
\hline V9000 & -3.127 & -3.172 & -0.045 \\
V1000 & 0 & 0 & - \\
V2000 & +1.534 & +1.588 & +0.054 \\
V3000 & +6.037 & +6.131 & +0.094 \\
V4000 & +6.944 & +7.024 & +0.080 \\
V5000 & +7.386 & +7.436 & +0.050 \\
V7000 & +14.821 & +14.881 & +0.060 \\
\hline
\end{tabular}

Table 6. Elevation errors from topographic measurements

A second step of control for the global result is the possibility to perform a roto-traslation of the ZEB point cloud in the established reference system. A residual error result can be defined as the quality of the positioning solution experimented in the proposed workflow. This can be done in twice solution, as Figure 6 shows. The rigid transformation can be executed with a manual picking points association on homologous points pairs, or with automatic calculation imposing the two coordinate tables and imposing the name correspondence between entities. In both cases, of course, the adjustment computation and residual evaluation delivered a final $\mathrm{RMSE}=0.06 \mathrm{~m} 3 \mathrm{D}$ vector, made of $\Delta \mathrm{x}=0.029 ; \Delta \mathrm{y}=0.035 ; \Delta \mathrm{z}=0.011$ spatial components.

\section{PURSUING DATA FUSION ISSUES: THE RGB ASSOCIATION WORKFLOW}

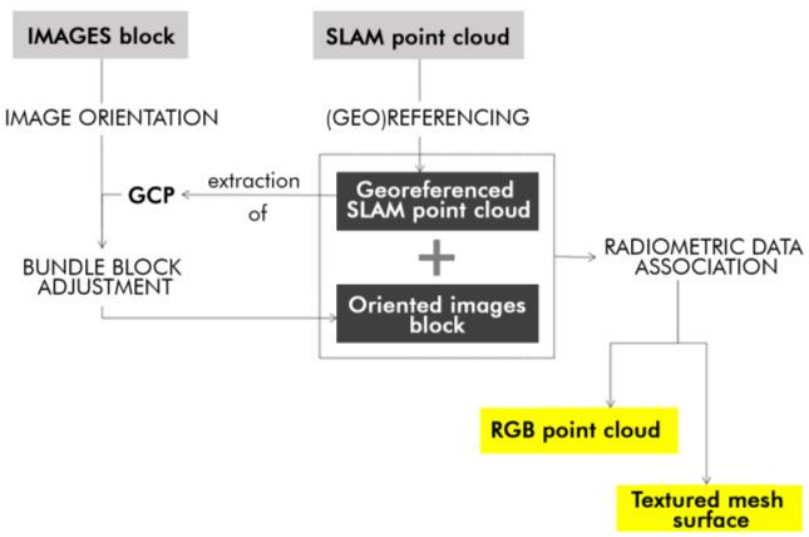

Figure 8 . The proposed pipeline to achieve the fusion between the RGB photogrammetric content and the SLAM point cloud

One of the focus of this research was connected with the data fusion between the SLAM dataset and the photogrammetric one. The main idea behind this test was to set up a rapid pipeline in order associate the radiometric data embedded in the photogrammetric dataset with the geometric information provided by the SLAM acquisition. The proposed pipeline is reported in the Figure 8 and will be briefly described.

The first steps of the pipeline are performed independently for the photogrammetric and the SLAM dataset and afterwards the processing of the two dataset is concluded in a single combined workflow. The images acquired were processed following the standard SfM (Structure from Motion) approach, at least for the first steps consisting in: the Tie Points extraction, the camera calibration, the image matching and the BBA (Bundle Block Adjustment) phases. The configuration of the image orientation block and the derived sparse cloud is reported in the Figure 9.

The image block orientation phase was solved using two different set of control points, as already reported: a set of points measured with a traditional topographic approach and a set of point which coordinates were extracted from the ZEB point cloud, after the georeferencig process described in section 3 and performed in parallel with the photogrammetric processing. A sample of the point extracted from the ZEB point cloud and used in the photogrammetric process is reported in

Figure 10. The RMSE (Root Mean Square Error) achieved following the two different strategies is reported in the Table 7.

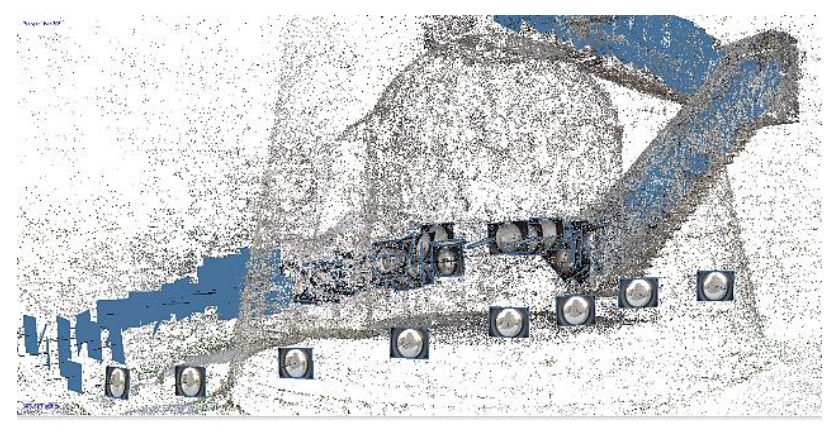

Figure 9. Orientation of the photogrammetric block. A view of the Tie Points sparse cloud and of the images position

\begin{tabular}{|l|ll|}
\hline \multirow{2}{*}{} & \multicolumn{2}{|c|}{ RMSE block adjustment } \\
\cline { 2 - 3 } & GCP & CP \\
\hline Topographic & $0.014 \mathrm{~m}$ & $0.022 \mathrm{~m}$ \\
ZEB-derived & $0.045 \mathrm{~m}$ & $0.057 \mathrm{~m}$ \\
\hline
\end{tabular}

Table 7. Comparison of RMS errors from the two blocks adjustment configurations

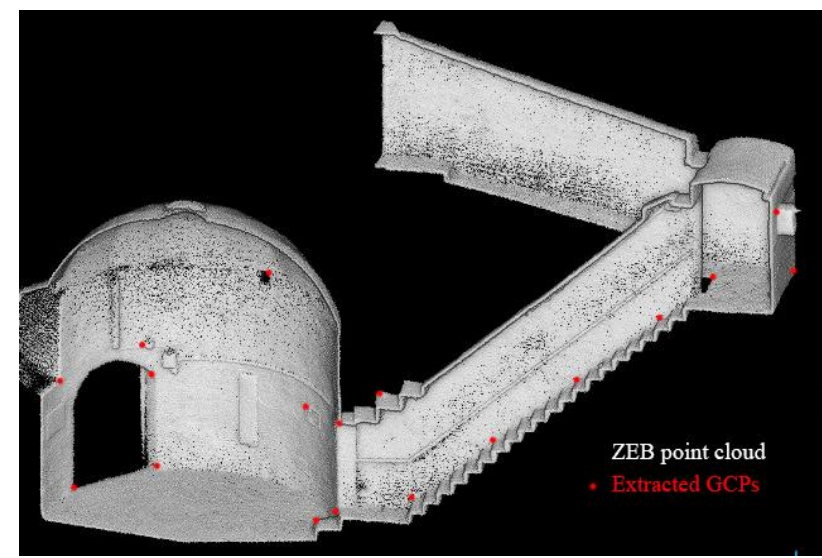

Figure 10. The portion of the SLAM point cloud used for testing the proposed approach, and e example of some of the control points extracted from the ZEB point cloud and used in the photogrammetric process

As it is possible to observe from the data reported in the table 7 , and as expected, the topographic method is returning more accurate results. Especially due to the fact that the point measured with the topographic approach consisted in pre-signalized well identifiable target, while the point extracted from the ZEB cloud were represented from natural feature with an intrinsic lower accuracy. However, these data are compatible with the intrinsic precision of the ZEB system and acceptable for the desired representation scale. In a second step of this pipeline, the SLAM point cloud can be imported in the selected software solution employed for the photogrammetric process, in this case Agisoft Metashape. Before completing this step, it is necessary to perform another preliminary step, i.e. computing the normals for the SLAM point cloud. Finally, it is possible to associate the RGB information of the DSRL images to the SLAM 3D model. This phase can be performed both on the point cloud, both after computing a mesh from the point cloud.

The proposed workflow is also based on the assumption of the unique reference system which both the data are processed and 
managed in: the Zeb-REVO point clouds, with the rigid rototraslation and georeferencing based on the computed reference points, previously measured as topographic vertices in the network, and the images block too, with bundle adjustment and GCPs. In fact, the image block orientation is based on a set of GCPs extracted from the georefereced ZEB point cloud and not based on individual topographic measures. However, the approach is metrically validated with the use of the same block aligned instead with topographic GCPs. The possibility to perform a direct radiometric association of data is exploited in order to provide a fusion-based 3D model with interesting potentials in terms of time-cost ration and enriched radiometric content, mostly in the 3D mesh surfaces, both of the tower entrance room and in a particular section of the "nottola" tunnel. Some first tests of the proposed pipeline were achieved on a portion of the 3D model, reported in the Figure 10.

\subsection{Radiometric data enrichment}

The normal computation process is not always a straightforward operation, especially when working with complex and articulated dataset such as the one of the F.I.N.E benchmark employed in this test. Several tests were performed to complete this operation using two different opensource software solution: Meshlab and CloudCompare. In both the software a first automatic computation of the normals data was performed considering the whole SLAM point cloud, however some areas with rough errors in the computed normal were still present. A second approach was thus created and consisted in a segmentation of the point cloud in smaller portion in order to achieve a better control on this phase. An example of the process of normal computation is reported in the following Figure 11.

In both the software solution the normal are computed adopting a local strategy analysis based on the number of neighbourhood and assuming several strategies for normal orientation. After this phase the point cloud with the new computed normal can be imported inside the photogrammetric software interface and can be further processed to generate other products such as $3 \mathrm{D}$ polygonal models as reported in e Figure 13 .

If some problems occurred in the phase of normal computation these issues are clearly visible during the phases of point cloud colorization, mesh and texture generation, as reported in the following Figure 12 for the point cloud. On the other side if the process succeeded the results of these preliminary test was encouraging and it was possible to achieve in a rapid a not expensive way (also from the computational point of view), a 3D textured mesh, together with a correctly colorized point cloud, thank to the previously oriented photogrammetric images block, based on Zeb-based GCPs extraction. The possibility to work with different levels of triangulation details starting from the Zeb point cloud Figure 10 allows to deliver low-weighted 3D models (Figure 13 top images) between $3 \mathrm{Mb}$ (*.obj format) $+9 \mathrm{Mb}$ texture file, instead of (bottom images) a $34 \mathrm{Mb}$ model $+10 \mathrm{Mb}$ texture. The numbers of triangles in Figure 13 are 1:10 ration between the two different models, but with results almost comparable for the final appearance of the model with the textured mesh (right).

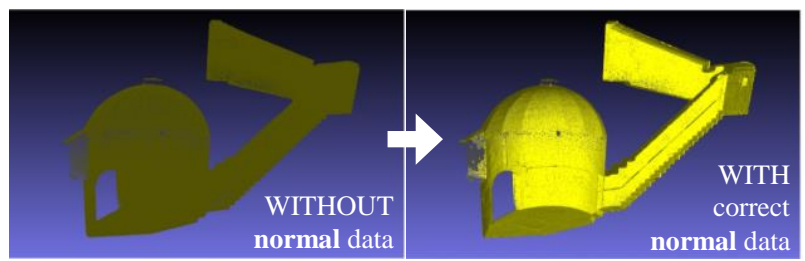

Figure 11. Normal computation process performed in the opensource software Meshlab. The original point cloud (left) and the point cloud after the normal computation (right)
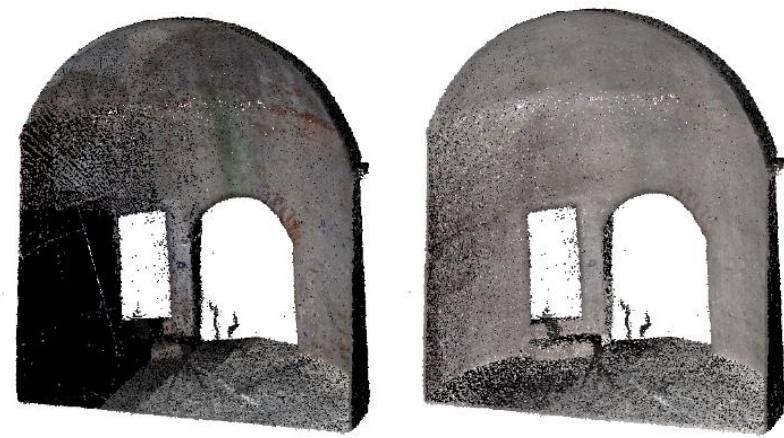

Figure 12. Example of some errors in the normals computation that lead to issues in the point cloud colorization

\section{CONCLUSIVE REMARKS}

The presented research for the SLAM-based mapping model enrichment by radiometric data offers some conclusive remarks to be discussed about the experimental workflow proposed for this benchmark. First of all, it is necessary to set some applicability boundaries according to the expected accuracy results and foresee the possible uses this kind of model, and the consequent required levels of detail. In the direction of possible solution for virtual and immersive modelling for dissemination purposes, a fluent navigation and management of $3 \mathrm{D}$ data is warmly recommended and

Figure 13. Sample of the point cloud A and mesh surface B after the import in the photogrammetric software interface. Visual comparison between high-detailed and low-detailed triangulated surface, according to three different views: wireframe (left); continua surface (centre); textured (right)
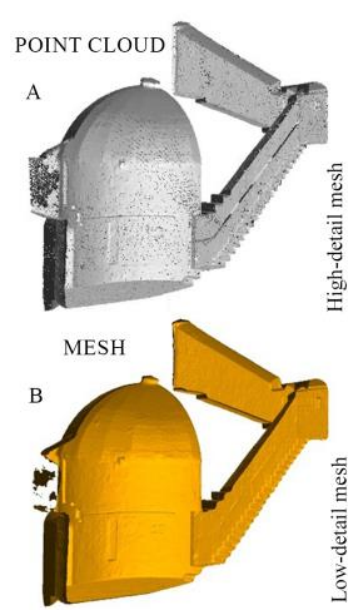
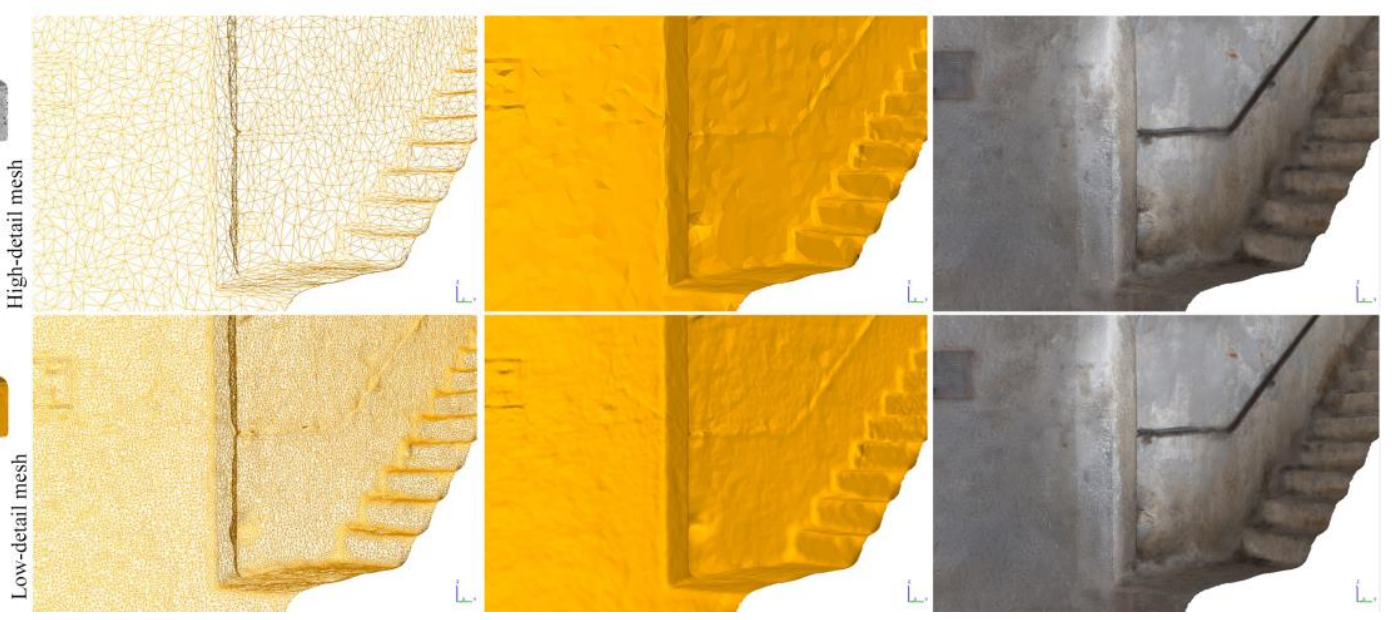
increasingly pursued, also applied to very large and complex area. Here the binding factor is the ease and continuity of data collection with portable and compact devices together with the possibility of having available a rapid and easy-to-use model where the maneuverability in terms of file weight, and the size of data is predominant compared to the research of the best possible accuracy. Moreover, the proposed (geo)positioning procedure for solving the problem of giving positioning attributes to the SLAM-based point cloud by MMS ZEB Revo RT resulted acceptable in terms of metric accuracy for the aforesaid aims. The new implementation allows registering reference points and relate the $3 \mathrm{D}$ mapping to the global reference system, with a controlled accuracy, at the moment, of few centimetres ( 1:100/1:200 scale)

What is more are the new perspectives in the radiometry data enrichment and HQ textures for SLAM-based point clouds by a novel approach of data fusion. Interesting potentials are offered by a proposed stand-alone fusion-based workflow in which the integration of fisheye photogrammetry and ranging methods contribute to overcome, if necessary, time-spending measurements operation and reduce topographic measurements actions. The possibility to use (geo)referenced SLAM-based point cloud for the image block orientation is successfully presented with the tolerance of the aforementioned accuracy pursued for digital models navigation. It has been flanked by a data-fusion in radiometric data attribute. In conclusion, the overall workflow for extensive and complex object modelling by SLAM-based mapping has proved to be a satisfactory approach despite some steps are currently under implementation. The mobile mapping system recommends good performances in the SLAM-based mapping of narrow and articulated spaces, allowing to obtain, parallelly, a complete survey of the indoor/outdoor area with controlled accuracy. In fact, the remarkable advantages are, overall, the portability/manoeuvrability and weight, the verified accuracy in enclosed/indoor spaces of few centimetres and in outdoor spaces of about a ten of centimetres. However, open issues related to the proposed pipeline will be, overall, a more automatic RGB data association implementation as time-consuming operation, and an optimization pipeline of the coherent normal computation for ZEB-based 3D mesh according to multiple and complex surfaces as the ones considered in this dataset.

\section{ACKNOWLEDGEMENTS}

We are grateful to F. Remondino and F. Fassi, together with their research group, for involving us and our study on SLAM based clouds assessment in the Fine benchmark proposed at the 3DARCH2019 conference (http://www.3d-arch.org/FINEbenchmark.pdf)

\section{REFERENCES}

Bakuła, K., Mills, J. P., \& Remondino, F., 2019. A review of benchmarking in photogrammetry and remote sensing. Int. Arch. Photogramm. Remote Sens. Spatial Inf. Sci., XLII-1/W2, 1-8.

Barazzetti, L., Previtali, M., \& Roncoroni, F., 2017. 3D modelling with the samsung gear 360. Int. Arch. Photogramm. Remote Sens. Spatial Inf. Sci., XLII-2, 85-90).

Bosse, M., Zlot, R., \& Flick, P., 2012. Zebedee: Design of a Spring-Mounted 3-D Range Sensor with Application to Mobile Mapping. IEEE Transactions on Robotics, 28(5), 1104-1119.

Cadge, S., 2016. Welcome to the ZEB REVOlution. GEOmedia, $22-26$.

Calantropio, A., Patrucco, G., Sammartano, G., \& Teppati Losè, L., 2018. Low-cost sensors for rapid mapping of cultural heritage: first tests using a COTS Steadicamera. Applied Geomatics, 10(1), 31-45.
Chiabrando, F., Naretto, M., Sammartano, G., Sambuelli, L., Spanò, A. T., \& Teppati Losè, L., 2017. The 4Dilan Project (4Th Dimension in Landscape and Artifacts Analyses). Int. Arch. Photogramm. Remote Sens. Spatial Inf. Sci., XLII-5/W1, 227234.

Chiabrando, F., Sammartano, G., Spanò, A., \& Spreafico, A., 2019. Hybrid 3D Models: When Geomatics Innovations Meet Extensive Built Heritage Complexes. ISPRS International Journal of Geo-Information, 8(3), 124.

Chiabrando, F., Sammartano, G., \& Spanò, A. T., 2017. A comparison among different optimization levels in 3D multisensor models. A test case in emergency context: 2016 Italian Earthquake. Int. Arch. Photogramm. Remote Sens. Spatial Inf. Sci., XLII-2/W3(March), 155-162.

Dewez, T. J. B., Yart, S., Thuon, Y., Pannet, P., \& Plat, E., 2017. Towards cavity-collapse hazard maps with Zeb-Revo handheld laser scanner point clouds. The Photogrammetric Record, 32(160), 354-376.

di Filippo, A., Sánchez-Aparicio, L., Barba, S., Martín-Jiménez, J., Mora, R., \& González Aguilera, D., 2018. Use of a Wearable Mobile Laser System in Seamless Indoor 3D Mapping of a Complex Historical Site. Remote Sensing, 10(12), 1897.

Eyre, M., Wetherelt, A., \& Coggan, J., 2016. Evaluation of automated underground mapping solutions for mining and civil engineering applications. Journal of Applied Remote Sensing, 10(4), 046011.

Mandelli, A., Fassi, F., Perfetti, L., \& Polari, C., 2017. Testing different survey techniques to model architectonic narrow spaces. Int. Arch. Photogramm. Remote Sens. Spatial Inf. Sci., XLII-2/W5, 505-511.

Nocerino, E., Menna, F., Remondino, F., Toschi, I., \& Rodríguez-Gonzálvez, P., 2017. Investigation of indoor and outdoor performance of two portable mobile mapping systems. In F. Remondino \& M. R. Shortis (Eds.), Videometrics, Range Imaging, and Applications XIV, edited, SPIE 2017, 103320I).

Perfetti, L., Polari, C., \& Fassi, F., 2018. Fisheye multi-camera system calibration for surveying narrow and complex architectures. Int. Arch. Photogramm. Remote Sens. Spatial Inf. Sci., XLII-2, 877-883

Rodríguez-Gonzálvez, P., Nocerino, E., Menna, F., Minto, S., \& Remondino, F., 2015. 3D surveying \& modeling of underground passages in wwi fortifications. Int. Arch. Photogramm. Remote Sens. Spatial Inf. Sci., XL-5/W4, 17-24.

Sammartano, G., \& Spanò, A., 2018. Point clouds by SLAMbased mobile mapping systems: accuracy and geometric content validation in multisensor survey and stand-alone acquisition. Applied Geomatics, 10(4), 317-339.

Søren Riisgaard, \& Morten Rufus Blas., 2005). SLAM for Dummies. A Tutorial Approach to Simultaneous Localization and Mapping. MIT OCW.

Sun, Z., \& Zhang, Y., 2019. Accuracy Evaluation of Videogrammetry Using A Low-Cost Spherical Camera for Narrow Architectural Heritage: An Observational Study with Variable Baselines and Blur Filters. Sensors, 19(3), 496.

Tucci, G., Visintini, D., Bonora, V., \& Parisi, E., 2018a. Examination of Indoor Mobile Mapping Systems in a Diversified Internal/External Test Field. Applied Sciences, 8(3), 401. 